\title{
A Novel Strategy to Improve the Performance of Speed And the Current loops for Servo Control
}

\section{QiongLi ${ }^{1}$}

State Grid Jiangxi Electric Power Research Institute

Nanchang, 330096, China

E-mail: powerdsp3390163.com

\section{ShengjianLi}

State Grid Jiangxi Electric Power Research Institute Nanchang, 330096, China

E-mail: powerdsp3390163.com

\section{Yan Liu}

State Grid Jiangxi Electric Power Research Institute Nanchang, 330096, China

E-mail: powerdsp3390163.com

\section{Yang Zou}

State Grid Jiangxi Electric Power Research Institute Nanchang, 330096,China

E-mail: powerdsp3390163.com

\begin{abstract}
The classical PI controllers are widely used in servo speed-loop and current-loop control structures because of their simple algorithm and stability.The corresponding PI control parametersaffect the performance of the loop responses. We always expect to expedite the response by improving Kp,but too largeKp will not only improve the speed and current responses but also bring oscillations and overshoots; thus the value of $\mathrm{Kp}$ is limited.The paper presents a new method to reduce the oscillations and overshoots for speed and current loops by means of low-order observers. The observers are used to extract the oscillation signals as compensation. The principle of the proposed method is explained and the rules of important controller parameters are described.Simulation and experiments are conducted to prove the advantage of the proposed strategy.Both the results have verifiedthe validity of the new strategy.
\end{abstract}

CENet2017

22-23 July 2017

Shanghai, China

\section{${ }^{1}$ Speaker}




\section{Introduction}

The speed-loop and the current-loop are the basic loops in PMSM(permanent magnet synchronous motor) control systems. The performance of the two loops plays a key role in the characteristics of the wholecontrol systems. As the PMSM drivers are extensively applied in the industrial fields, it is very significant to improve their performance. The PI controller is generally regarded as one of the most practical controllers in the control systems due to its simplicity. Generally, PI controller parameters are set to achieve the fastest step response without lessening its stability. As it's known to all, the increasing $K_{p}$ is the most direct way to improve the response, but it may also bring about oscillations and even make the system unstable if $K_{p}$ is too large. In order to improve the responses of controllers and suppress oscillations at the same time, many strategies have been presented.

Chengbin Ma discussed different PID structures, the modified IP(m-IP), the integralproportional (IP), and the modified integral-proportional-derivative (m-IPD) and then features of these PID controllers were compared[1-2]. He pointed out that both IP controllers and m-IPD controllers could reduce the oscillations, but the PID controller parameters must be set according to the inertia ration tolimit its use. The author figured out a method to obtain the balance between the magnitude margin loss, the phase margin loss and the strength of oscillation robustness by a fractional order controller[3]. Wen Li used a PI fuzzy controller with a disturbance observer for oscillation reducing[4]. Firstly, a PI fuzzy controller was adapted to ensure the expected static accuracy and then the disturbance observer was used to obtain the compensation signal. A systematic wavelet control strategy was applied to improve the rapid of speed command responses [5]. The results verified the efficiency of the algorithm, but the tests were carried out for the micro PMSM only.

This paper proposes a method to improve the speed response and current response. Firstly, a current state observer is built to overcome the phase delay in current calculations. Then the observed current and practical feedback current are used to extract oscillation signal to further reduce the overshoot; for the speed-loop control, an observer is also used to extract speed oscillation information as compensation of speed feedback. The advantage of the new control algorithm has beenproved by both the simulation and the experiments results.

\section{Current and Speed Oscillation SuppressionMethod}

Usually, the PI controller for current and speed loop is designed to remove the transfer function poles. According to the response of II-system, as the growth of proportional gain KP, the current and speed dynamic response are faster. At the same time, it brings overshoot and oscillation[6]. Fig.1 describes the reason whythe frequency response of speed loop transfer function will have a obvious peak as Kp grows.

For the design of current and speed loop, the shorter settling time and faster response is desired. In order to improve the rapid of current response and shorten settling time, the oscillation should be suppressed. Here, the currentstate observer and speed oscillation extracting structure are combined to achieve the objective.

Firstly, we discuss the current oscillation method. We know that the peak of magnitude gains leads to the oscillation of the control loop, so if we can reduce the peak, we can suppress the oscillation. Here, we adapt the current observer and the current compensation structure to achieve the current oscillation suppression. According to electrical subsystem of the PMSM, we can obtain the current state in Equation 2.1. 


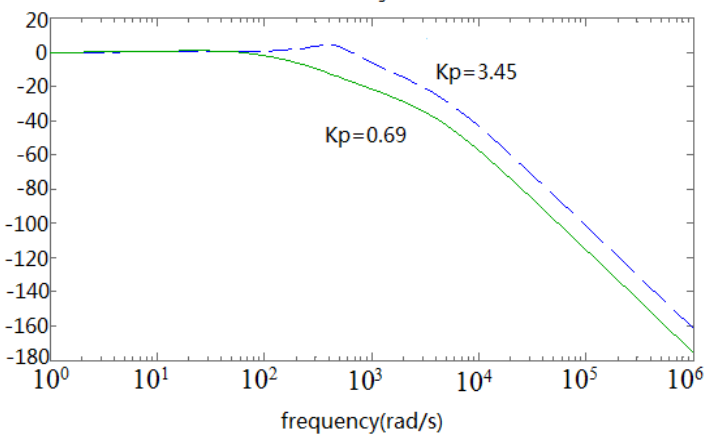

Figure 1:The Bode Diagrams of Speed Loop Transfer Function with Different Kp

$$
\left[\begin{array}{c}
\dot{i_{d}} \\
\dot{i_{q}}
\end{array}\right]=\left[\begin{array}{cc}
-\frac{R}{L} & \omega_{e} \\
-\omega_{e} & -\frac{R}{L}
\end{array}\right]\left[\begin{array}{l}
i_{d} \\
i_{q}
\end{array}\right]+\left[\begin{array}{ll}
\frac{1}{L} & 0 \\
0 & \frac{1}{L}
\end{array}\right]\left[\begin{array}{l}
u_{d} \\
u_{q}
\end{array}\right]+\left[\begin{array}{c}
0 \\
-\frac{\varphi_{f}}{L} \omega_{e}
\end{array}\right]
$$

Then the state observer can be defined as:

$$
\left[\begin{array}{c}
\dot{\hat{i}} \\
\dot{i_{d}} \\
\hat{i_{q}}
\end{array}\right]=\left[\begin{array}{cc}
-\frac{R}{L} & \omega_{e} \\
-\omega_{e} & -\frac{R}{L}
\end{array}\right]\left[\begin{array}{c}
\hat{i_{d}} \\
\hat{i_{q}}
\end{array}\right]+\left[\begin{array}{cc}
\frac{1}{L} & 0 \\
0 & \frac{1}{L}
\end{array}\right]\left[\begin{array}{l}
u_{d} \\
u_{q}
\end{array}\right]+\left[\begin{array}{c}
0 \\
-\frac{\varphi_{f}}{L} \omega_{e}
\end{array}\right]+\left[\begin{array}{cc}
l_{1} & 0 \\
0 & l_{2}
\end{array}\right]\left[\begin{array}{c}
i_{d}-\hat{i_{d}} \\
i_{q}-\hat{i_{q}}
\end{array}\right]
$$

Where and are the observed variables, is the electrical angle speed, and is the adjustment coefficient.

The characteristic polynomial of observer can be written as:

$$
f(\lambda)=|\lambda I-(A-K C)|
$$

Where, ,. Assumethat the desired eigenvalues be, then the designs of ,should satisfy the following equation:

$$
f\left(\lambda^{*}\right)=f(\lambda)=\left(\lambda-\lambda_{1}^{*}\right)\left(\lambda-\lambda_{2}^{*}\right)
$$

The observed current, is used as the feedback of oscillation extracting structure, in the paper, we proposed a structure like Fig. 2 to compensate the current oscillation. Low-pass filter and high-pass filter are used to extract the oscillation signals. This is a simple structure but has been verified to be a practical and saving soft recourse. , are thelow-pass filter cutoff frequency and high-pass filtercutoff frequency respectively. We can get the current oscillation signal by tuning, , and the current feedback is redefined as. The output is used as the feedback for current-loop, so the current oscillation can be removed in this way.

The previous discussion concernsthe current oscillation method. To decrease the speed oscillations, we present a control structure like Fig. 3, where $\mathrm{k}_{\text {vib }}$ is the speed vibration observer parameter;, $\mathrm{k}_{\mathrm{j}} \mathrm{andk}_{\mathrm{lf}} \mathrm{refer}$ tothe measurement noise, the reciprocal value of $\mathrm{J}_{\mathrm{m}}$ and the bandwidth of low-pass filter respectively. LF is a low-pass filter. In the previous section, we have known the influence of $\mathrm{Kp}$ on the performance of system response, so the point to reduce vibration caused by strong $K_{p}$ is to suppress the magnitude gain at oscillation point. Thenext part will explain the parameters design to realize the concept. 


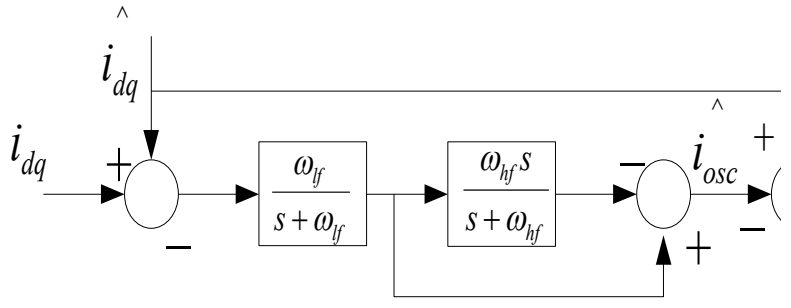

Figure 2:The Structure of Current

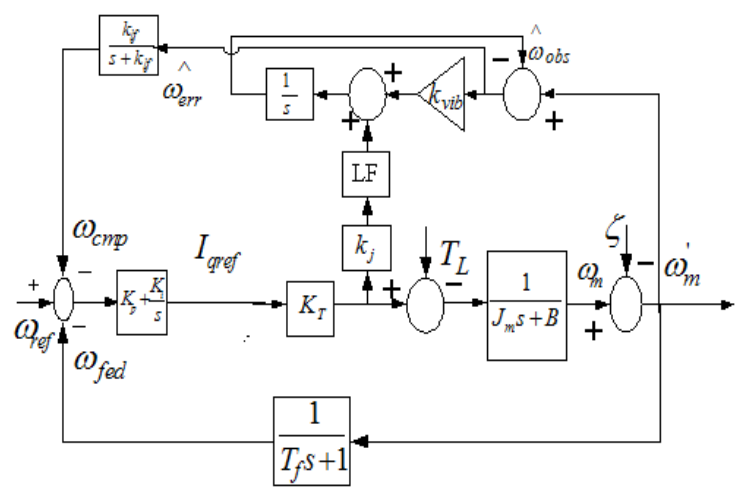

Figure 3:The Proposed Method's Structure Oscillation Suppression

\section{Parameters Design of the Speed Oscillation Suppression}

1) Oscillation frequency analysis

In this paper, in order to study the speed error spectrum, discrete Fourier transform (DFT) was used. Thenthe PSD(power spectrum density) would be obtained based on the FFT output. The oscillation frequency corresponds to the highest PSD.

2) The design of and

The oscillation frequency is known by using FFT.Assume to reduce adjustable parameter,then we reconsider the transfer function $\mathrm{G}_{\mathrm{rm}}(\mathrm{s})=/$ with(the speed oscillation compensation) and suppose $T_{f}=0, \mathrm{~B}=0$ forsimplicity,so $\mathrm{G}_{\mathrm{rm}}(\mathrm{s})$ can be obtained inEqu. 3.1.

$$
\begin{aligned}
& G_{2}=K_{T}\left(K_{p}+\frac{K_{i}}{s}\right) \frac{1}{J_{m} s} \\
& H_{2}=1+\frac{s}{s+\omega_{v i b}} \frac{k_{l f}}{s+k_{l f}} \\
& G_{r m}(s)=\frac{\omega_{m}(s)}{\omega_{r e f}(s)}=\frac{G_{2}}{1+G_{2} H_{2}}
\end{aligned}
$$

Then the characteristic polynomial in the speed closed-loop is shown as follows:

$$
\begin{aligned}
\Delta P(s)=1+G_{2} H_{2} & =a_{4} s^{4}+a_{3} s^{3}+a_{2} s^{2}+a_{1} s+a_{0} \\
a_{4}= & J_{m} \\
a_{3}= & J_{m}\left(\omega_{v i b}+k_{l f}\right)+K_{T} K_{p} \\
a_{2}= & K_{i} K_{T}+K_{T} K_{p}\left(\omega_{v i b}+2 k_{l f}\right) \\
& +J_{m} \omega_{v i b} k_{l f} \\
a_{1}= & K_{T} K_{p} \omega_{v i b} k_{l f}+K_{i} K_{T}\left(\omega_{v i b}+2 k_{l f}\right) \\
a_{0}= & K_{i} K_{T} \omega_{v i b} k_{l f}
\end{aligned}
$$


Additionally, the numerator polynomial is writen as:

$$
\begin{aligned}
\Delta N(s) & =K_{p} K_{T} s^{3}+\left[K_{p} K_{T}\left(\omega_{v i b}+K_{l f}\right)+K_{i} K_{T}\right] s^{2} \\
& +\left[K_{p} K_{T} \omega_{v i b} k_{l f}+K_{i} K_{T}\left(\omega_{v i b}+k_{l f}\right)\right] s+K_{i} K_{T} \omega_{v i b} k_{l f}
\end{aligned}
$$

To cancel the peak of $\mathrm{G}_{\mathrm{rm}}(\mathrm{s})$ at the oscillation frequency, we hope $\left|\mathrm{G}_{\mathrm{rm}}(\mathrm{s})\right|=1$ at the oscillation point. Thus we could deduce the following equations:

$$
\begin{aligned}
& \left|\frac{j b_{1}+b_{0}}{j a_{1}+a_{0}}\right|=1 \\
& a_{1}=\left[-\left(J_{m}\left(k_{l f}+\omega_{v i b}\right)+K_{p} K_{T}\right) \omega_{v i b}^{2}+K_{T} K_{p} \omega_{v i b} k_{l f}+K_{T} K_{i}\left(\omega_{v i b}+2 k_{l f}\right)\right] \omega_{v i b} \\
& a_{0}=K_{T} K_{i} \omega_{v i b} k_{l f}-\left(J_{m} \omega_{v i b} k_{l f}+K_{T} K_{i}+K_{p} K_{T}\left(\omega_{v i b}+2 k_{l f}\right)\right) \omega_{v i b}^{2} \\
& b_{1}=\left[-K_{p} K_{T} \omega_{v i b}^{2}+K_{T} K_{p} \omega_{v i b} k_{l f}+K_{T} K_{i}\left(\omega_{v i b}+k_{l f}\right)\right] \omega_{v i b} \\
& b_{0}=K_{T} K_{i} \omega_{v i b} k_{l f}-\left[K_{T} K_{i}+K_{p} K_{T}\left(\omega_{v i b}+k_{l f}\right)\right] \omega_{v i b}^{2}
\end{aligned}
$$

As we know is equal to nearly based on previous section, it can be seen. Since Ki equals to , we can simplify the above equations. Support, then Equ.(3.4) can be derived:

$$
\begin{aligned}
& a_{1}=K_{T} K_{p} \frac{n+1}{2} \\
& a_{0}=-K_{T} K_{p} \frac{n+1}{2} \\
& b_{1}=K_{T} K_{p}\left(n-\frac{3}{2}\right) \\
& b_{0}=-K_{T} K_{p} \frac{3}{2} \\
& \frac{\left(\frac{1}{2}(n+1)\right)^{2}+\left(-\frac{1}{2}(n+1)\right)^{2}}{\left(n-\frac{3}{2}\right)^{2}+\left(\frac{3}{2}\right)^{2}}=1
\end{aligned}
$$

It is obvious both and are the solutions of Equ. 3.4, but in ordreto ensure the phase marginat the oscillation point, must be satisfiedsince, therefore is the only solution. In conclusion:

$$
k_{l f}=6.8 \omega_{v i b}
$$

4)The oscillation suppression theory of the proposed strategy

We will discuss the oscillation suppression reason by analyzing the speed reference response with the proposed strategy and the traditional PI controller respectively.

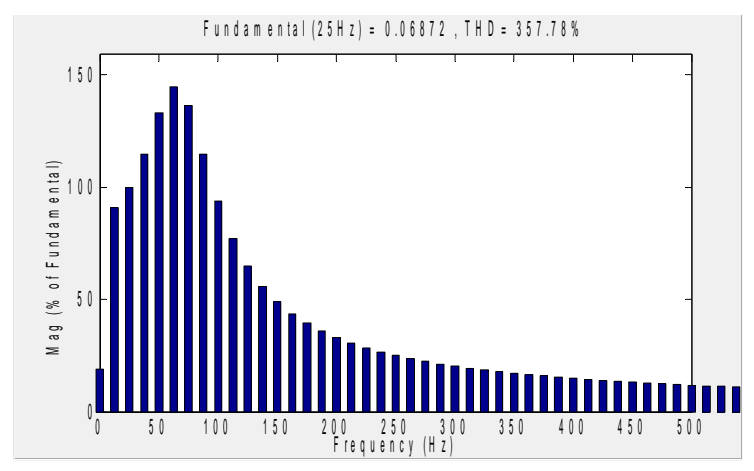

Figure 4:The Frequency Spectrum

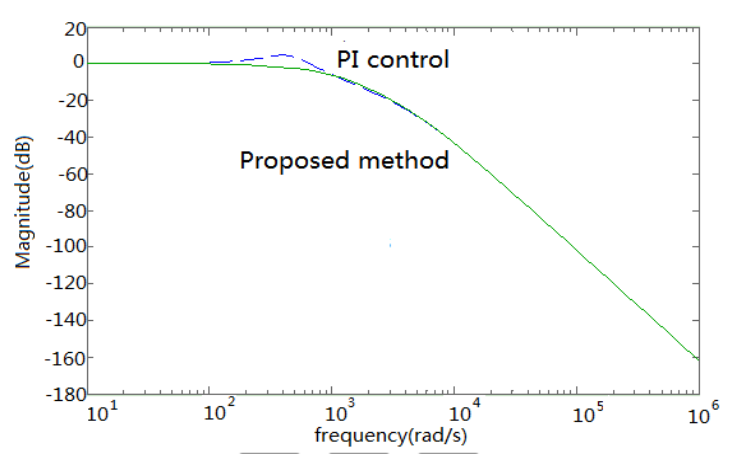

Figure 5:The Frequency Response of Speed Error of Speed Command 


\begin{tabular}{ccc}
\hline parameters & values & unit \\
\hline$J_{m}$ & 0.008 & $\mathrm{Kg} \cdot \mathrm{m}^{2}$ \\
$R_{s}$ & 2.870 & \\
$L_{q}$ & 0.0085 & $\mathrm{H}$ \\
$K_{T}$ & 1.0 & $\mathrm{Nms}$ \\
$T_{f}$ & 2.0 & $\mathrm{~ms}$ \\
\hline
\end{tabular}

Table 1:The Simulation Parameters

Table I describesthe simulation parameters. The frequency spectrum of is obtained by performing FFT as Fig. 4a. The other parameters are designed by previous proposed principles. Based on the information, the frequency responses of speed reference with the proposed strategy and traditional PI controller can be plotted as Fig. 4b; on this basis, we can find the magnitude gainwith PI controlleralgorithm is much stronger in comparison with the proposed strategy at oscillation frequency. The magnitude gain is around zerowith the presentstrategywhich satisfies the requirement discussed in parameters design section. Thus the idea of lowering the oscillation peak to reduce oscillation can be realized byusing the proposed method. The speed reference response of proposed method performs better than that of PI control.

\section{The Experiment Results}

In order to testify the effectiveness of the present strategy, Fig. 5a-5b describes the control structure of the method and the experiment platform. It consists of a PC for saving experimental data, a servo driver and a PMSM. Both the present strategy and the classical PI control method are tried under the same condition.

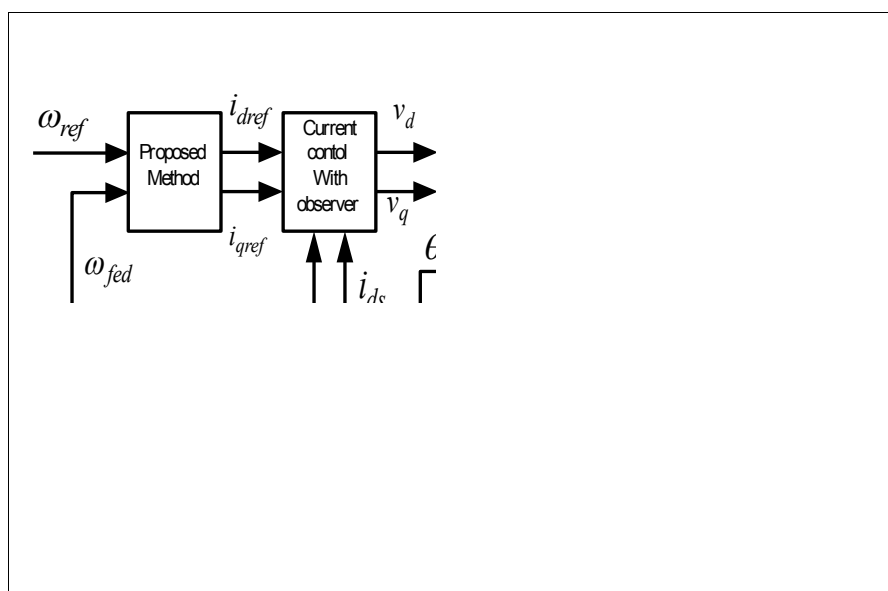

Figure 6: Software Structure

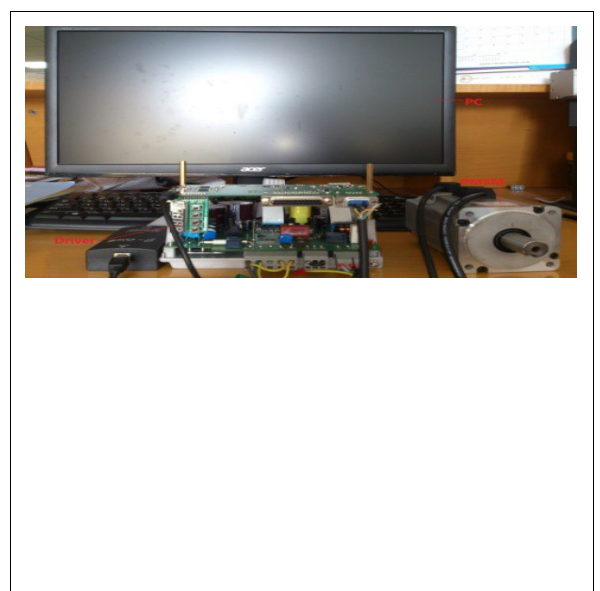

Figure7: Experimental Platform

Firstly, we verify the effect of current observer. Fig. 6 shows the current step response with and without current observer. We can see the overshoot and oscillation is suppressed by using the current observer. The settling time without current observer is about $0.85 \mathrm{~ms}$ in comparison with $0.6 \mathrm{~ms}$ with current observer. Also, the current feedback without observer has $15 \%$ overshoot, while the overshoot is suppressed with current observer. The current oscillation is shown in Fig. 6btoo.We can see that the current oscillation is similar to the overshoot without current observer, it is removed by proposed method, so the current feedback with observer has no overshoot. Therefor, the current observer is available. 


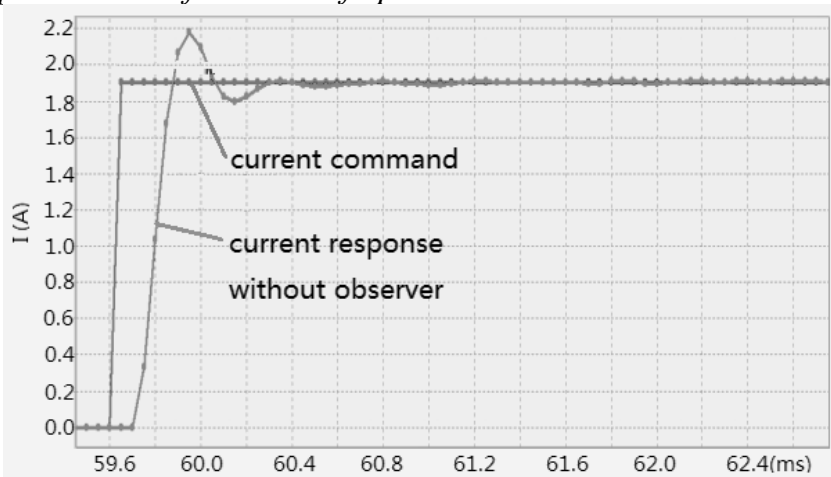

Figure 8: Current Response without Observer

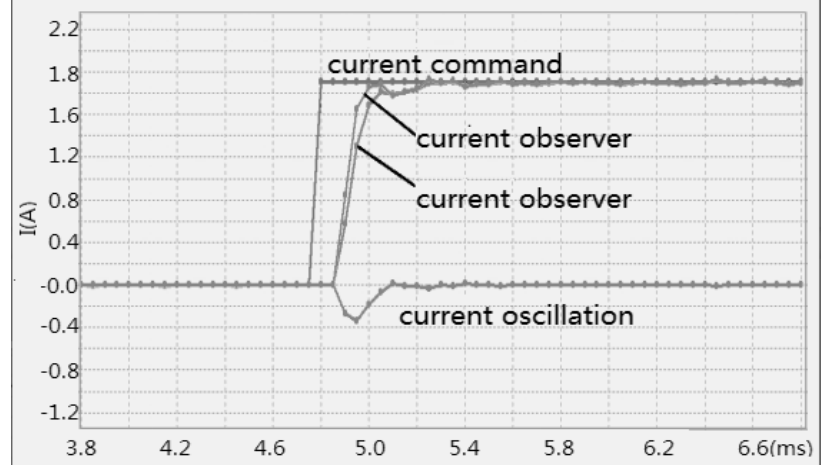

Figure 9: Current Responsewith Observer

\begin{tabular}{cccc}
\hline \multirow{2}{*}{ items } & \multicolumn{2}{c}{ overshoot } & $\begin{array}{c}\text { Vibration } \\
\text { duration }\end{array}$ \\
\cline { 2 - 4 } & PI control & $\begin{array}{c}\text { Proposed } \\
\text { method }\end{array}$ & PI control \\
\hline $\mathrm{Kp}=3.45$ & $20 \%$ & $10 \%$ & $4 \mathrm{~ms}$ \\
$\mathrm{Kp}=5.01$ & $55 \%$ & $20 \%$ & $7 \mathrm{~ms}$ \\
$\mathrm{Kp}=8.09$ & $\begin{array}{c}\text { Out of } \\
\text { control }\end{array}$ & $40 \%$ & $18.5 \mathrm{~ms}$ \\
\hline
\end{tabular}

Table 2: The Speed Response with PI Controller and Present Strategy

Next, we analyze the speed responses. The results are shown in Fig. 7-9. Table 2 concludes the difference under the two control methods. The data show that the speed response performs worse as $K_{p}$ grows under PI control, the overshoot is bigger and the vibration lasts longer. However, the speed response still performs wellwiththe propose method. The overshoot is smaller and the vibration is much shorter. Thus, it is obvious that the proposed method is available in oscillation suppression.

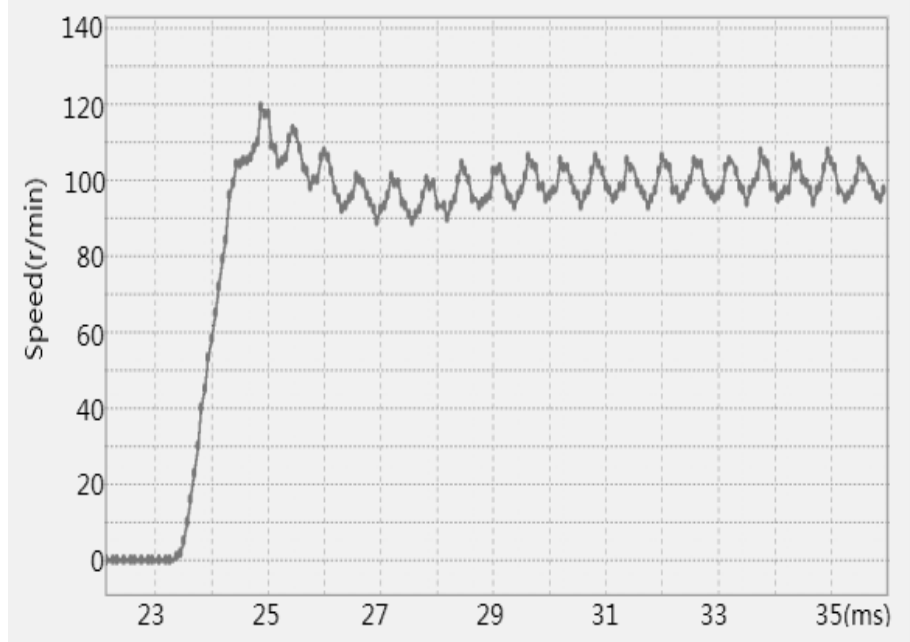

(a) PI control 
(b)the present strategy

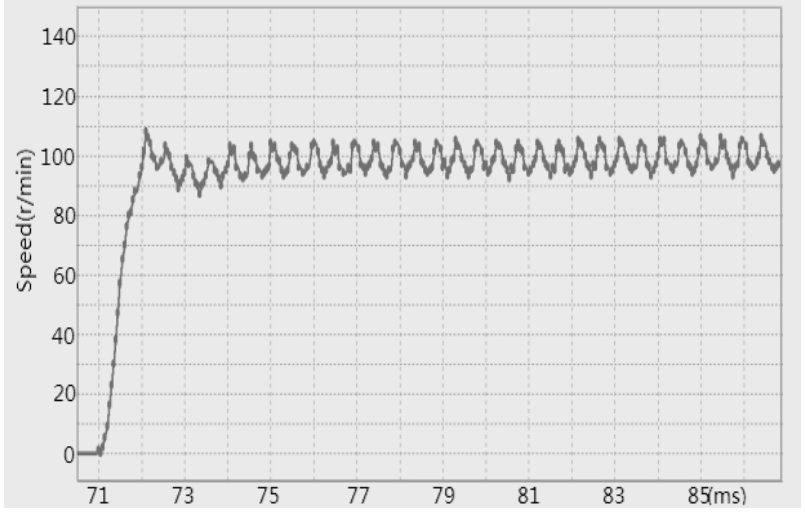

Figure 10: Speed Step Response with $K_{p}=3.45 \mathrm{rad} / \mathrm{s}$

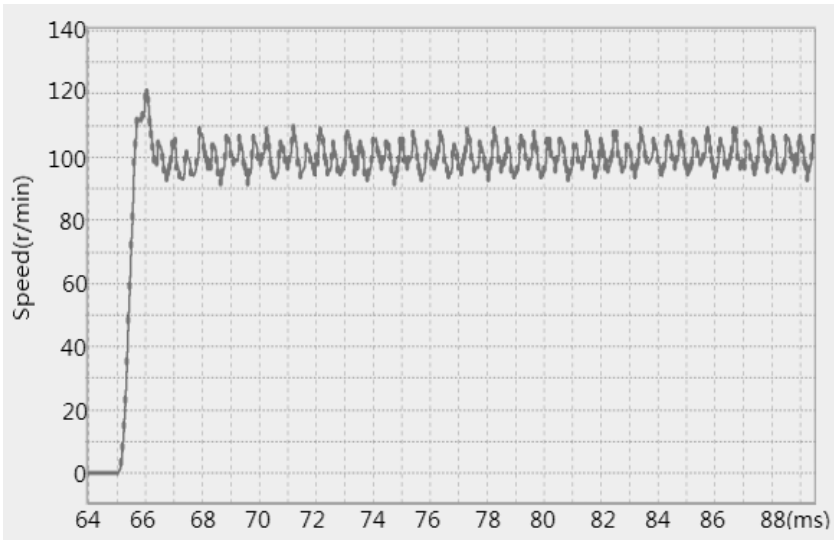

(a) PI control

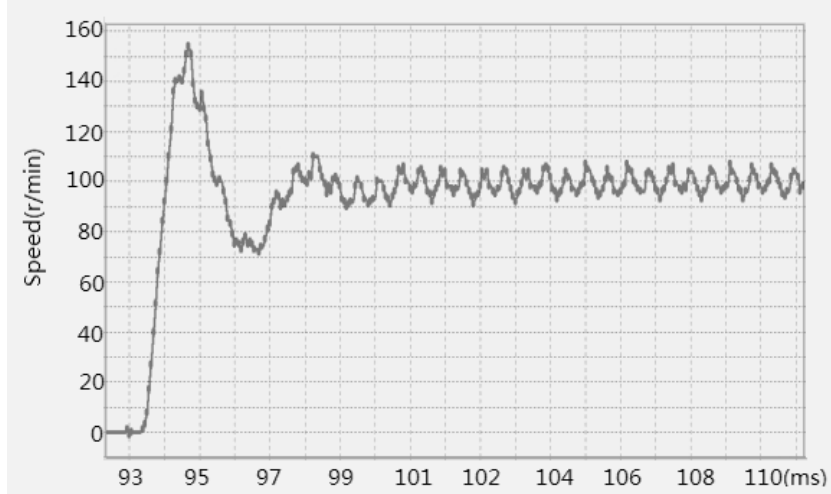

(b)The Present Strategy

Figure 11: Speed Step Response with $K_{p}=5.01 \mathrm{rad} / \mathrm{s}$

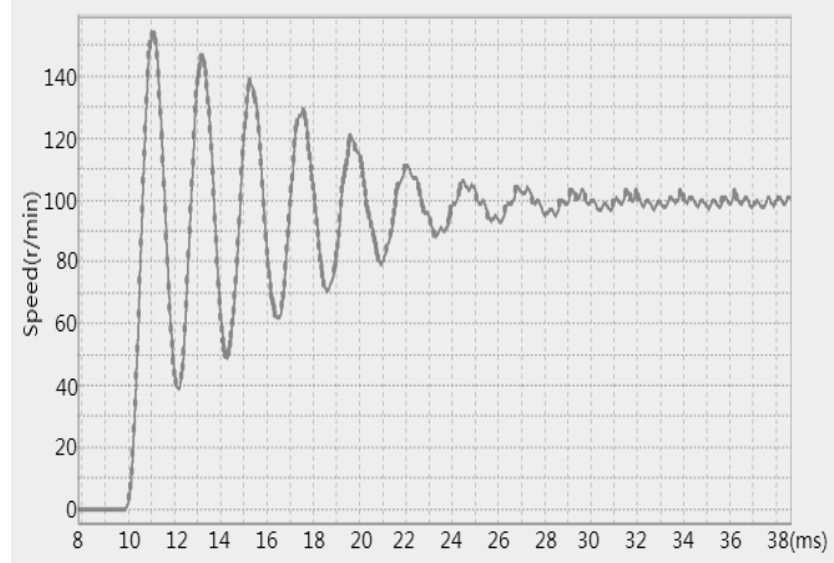

(a) PI control 


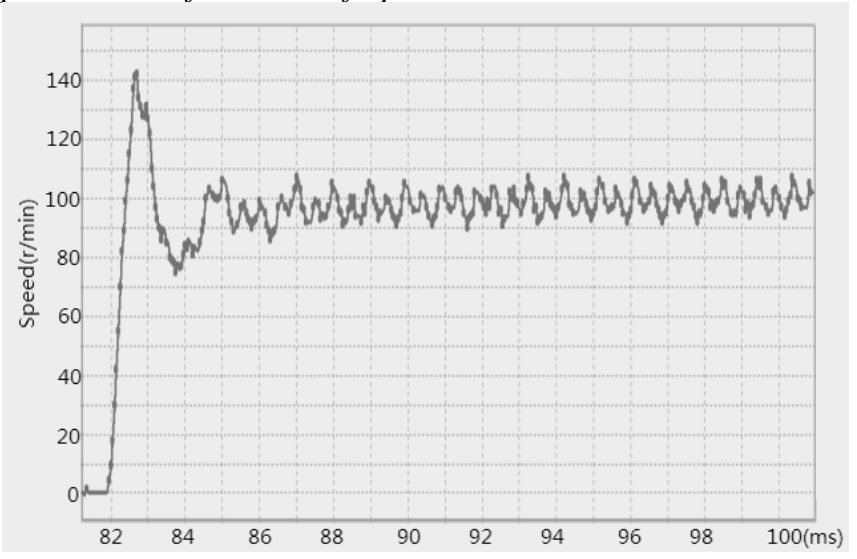

(b) The Present Strategy

Figure 12: Speed Step Response with $K_{p}=8.09 \mathrm{rad} / \mathrm{s}$

\section{Conclusion}

In this paper, we present a current observer to improve the current-loop performance to reduce the settling time and overshoot. The current-loop is the fundamental control in servo drive control systems. Then we analyze the reason of oscillation with high speed-loop gain and propose the oscillation reducing method on the basis of counteracting resonance peak at oscillation frequency. We use FFT to calculate the oscillation frequency and draw the oscillation by combining low-pass filter and speed observer as the compensation for the speed feedback. The design rules of controller parameters ensure the effect of control strategy. In this paper, we discuss the design rules in details and simplify the design specifications by observing the transfer functions. The frequency response diagrams of the traditional PI controller and the present strategy explain the key idea of oscillation suppression theoretically. The experiment results also verify that the present strategy has improved the performance of speed loop and current loop effectively. With the reducedcurrent oscillation and speed oscillation, we can get higher loop-gain to assist in the satisfication of the fast system response requirement.

\section{References}

[1] Chengbin Ma.,Junyi.Cao., etc. Polynomial-Method-Based Design of Low-order Controllers for TwoMass Systems. Industrial Electronics, IEEE Trans., 60(3):969-978(2013).

[2] Chengbin Ma., Hori Y.Backlash, Vibrationsuppress- ion in torsional system based on the fractional order-Q-filter of disturbance observer . Proc 8th IEEE Int. Workshop Adv. Motion contr., p. 577-582(2004).

[3]Chengbin Ma., Hori Y,The application backlash of fra-ctional order control to vibration suppression. Proc Conf. American Contr., p. 2901-2906(2004).

[4] Wen Li., Hori Y, . Vibration Suppression Using Sing- le Neuron-Based PI Fuzzy Controller and Fractional-order Disturbance Observer. Industrial Electronics, IEEE Trans., 54(1): 1117-126.

[5] Wan-Cheng Wang, Tian-Hua Liu, Kuang-Yao Fan. Design and implementation of a wavelet speed contr-oller with application to micro- permanent magnet synchronous motor drives. Electric Power Applica- tions, IET., 7(4):245-255(2013).

[6] Dong Heng., Hui Wang., Ke-yuan Huang. Design of PMSM Driver system Digital PI Adjuster Parameters. Electric Drive., 39(1):7-9(2009). 